\title{
An Improved Method for Determination of Refractive Index of Dielectric Films from Reflectance Spectrum by Using the Generalized Morse Wavelet
}

\author{
Erhan Tiryaki ${ }^{1}$, Özlem Kocahan ${ }^{2}$, Serhat Özder ${ }^{3}$ \\ ${ }^{1}$ Department of Physics, School of Graduate Studies, Canakkale Onsekiz Mart University, 17020, Canakkale, Turkey, \\ erhantiryaki@gmail.com \\ ${ }^{2}$ Department of Physics, Faculty of Arts and Sciences, Namik Kemal University, Tekirdag, 59030, Turkey \\ ${ }^{3}$ Department of Physics, Faculty of Arts and Sciences, Canakkale Onsekiz Mart University, Canakkale, 17020, Turkey
}

\begin{abstract}
The Generalized Morse Wavelet (GMW) algorithm was adapted to determine the refractive index of dielectric film from the reflectance spectrum. A theoretically generated reflectance spectrum in the range of 300-1200 nm wavelength was analyzed by the Continuous Wavelet Transform (CWT) and the refractive index dispersion was obtained by the mentioned method. In addition, a noisy reflectance spectrum was analyzed to show the advantages of the CWT method. Refractive index dispersions calculated by the Morlet and the Paul wavelet were compared to GMW at the end of the study.
\end{abstract}

Keywords: Dielectric film, refractive index, reflectance spectrum, continuous wavelet transform, generalized Morse wavelet.

\section{INTRODUCTION}

Precise measure of optical parameters such as refractive index and absorption coefficient has been the most significant subject for the production of optical-based devices in material science and industry. Therefore, many methods have been developed. Most common methods use both transmittance and reflectance spectrum of thin film to determine these optical parameters. The thickness and optical constants of absorbing thin films were determined from the transmittance spectrum via the envelope method [1], [2]. The envelope method was also applied to the reflectance spectrum to determine the optical constants [3], [4]. Abbe refractometer [5] and ellipsometry [6] are common systems to measure the refractive index. The CWT method is one of the most useful tools to analyze the time-dependent signal for obtaining frequency information. CWT provides analysis of the nonstationary signal, which is a widely used mathematical approach in data analysis such as signal and image processing [7]. Furthermore, it was enhanced to determine the optical parameters [8]-[10]. The determination of physical thickness (d) and refractive index (n) of dielectric films constitute an important role for the optoelectronic device and its applications [11], [12]. The thickness and the refractive index can be changed for optimization while designing thin films [12]. Since dielectric optical thin films have importance in optical fiber sensor technologies, optical characterization of these films is a critical issue [13]. It is convenient to use a self-supporting film surrounded by air on both sides and for normal lighting when they are optically tested. Optical tests of dielectric films can be performed with this proposed method. Mica, a birefringent material, which is one of the standard materials used as retardation plates [14], was used as a sample for the measurement of the refractive index since it is a natural substance and cleaves perfectly into the thin plate [15]. In that study, the generalized S-transform was used for the calculation of the refractive index from the transmittance spectrum. Here, we provide an alternative approach to determine the refractive index of dielectric film from the reflectance spectrum based on a theoretical method and a simulation study. Because the repetition frequency of the reflectance spectrum is related to optical thickness, it is also related to the refractive index.

In the CWT method, choosing the wavelet function is a very crucial point. Morlet wavelet is a commonly used one but it has a constant shape and there is no ability to control the analysis resolution. Conversely, Paul wavelet which has one degree of freedom, has the ability to control the resolution. Besides, GMW has an advantage with two degrees of freedom. These two parameters lead to more control on the resolution.

In this study, GMW was improved to obtain the refractive index of dielectric film from the reflectance spectrum. Also, 
the results obtained by GMW were compared with Morlet and Paul wavelets. In addition, a noisy reflectance spectrum was analyzed to show the advantages of the CWT method.

\section{SUBJECT \& METHODS}

A linearly polarized light (TE mod), shown in Fig.1., comes from the medium which has the refractive index $n_{1}$ and interacts with the dielectric film which has thickness $d$ and refractive index $n_{2}$ with the angle of incidence $\theta_{1}$, then it passes through with the angle of incidence $\theta_{2}$ from the dielectric film into the medium with refractive index $n_{3}$. The reflectance spectrum of the dielectric film given by equation (1) is obtained by using the electric and magnetic field components of the polarized light at the boundary I and II [16].

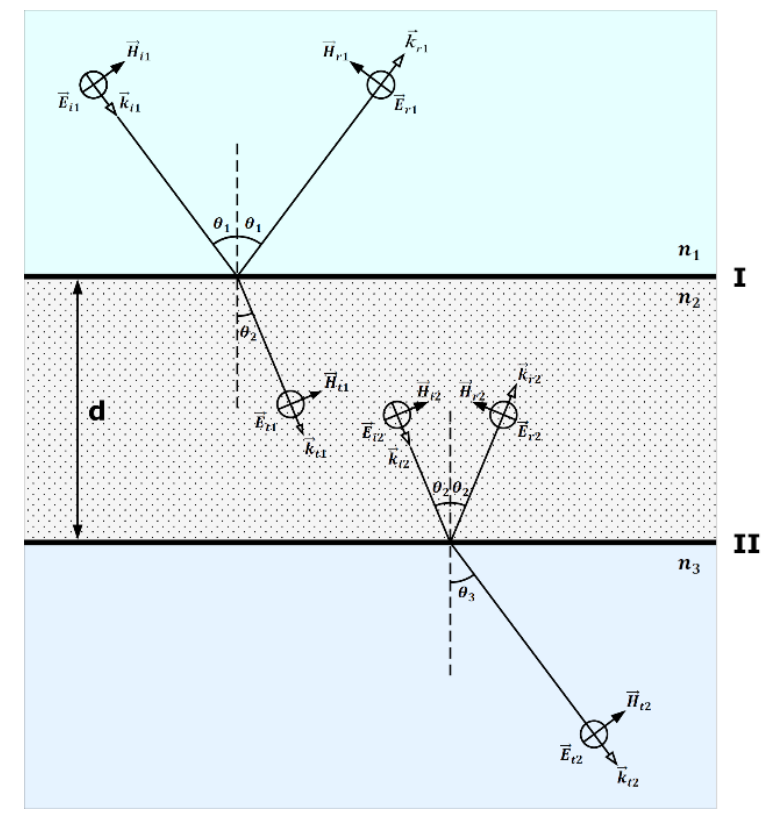

Fig.1. The movement of the polarized light (TE mode) throughout mediums which have refractive indexes $n_{1}, n_{2}$, and $n_{3}$. The state of fields which are electric field $\vec{E}$, magnetic field $\vec{H}$, and propagation vector $\vec{k}$ at the boundaries.

$$
\begin{gathered}
R\left(k_{0}\right)=r^{2}=\left[\begin{array}{cc}
\frac{\rho_{1} m_{11}+\rho_{1} \rho_{3} m_{12}-m_{21}-\rho_{3} m_{22}}{\rho_{1} m_{11}+\rho_{1} \rho_{3} m_{12}+m_{21}+\rho_{3} m_{22}}
\end{array}\right]^{2} \\
M=\left[\begin{array}{ll}
m_{11} & m_{12} \\
m_{21} & m_{22}
\end{array}\right]=\left[\begin{array}{cc}
\cos \left(h k_{0}\right) & \left(i \sin \left(k_{0} h\right) / \rho_{2}\right) \\
\left(i \sin \left(k_{0} h\right) \rho_{2}\right) & \cos \left(h k_{0}\right)
\end{array}\right] \quad(1 \mathrm{~b}) \\
\rho_{1}=\sqrt{\frac{\varepsilon_{0}}{\mu_{0}}} n_{1} \cos \left(\theta_{1}\right), \quad \rho_{2}=\sqrt{\frac{\varepsilon_{0}}{\mu_{0}}} n_{2} \cos \left(\theta_{2}\right), \quad \rho_{3}=\sqrt{\frac{\varepsilon_{0}}{\mu_{0}}} n_{3} \cos \left(\theta_{3}\right) \text { (1c) } \\
h=2 \pi n_{2} d \cos \left(\theta_{2}\right)
\end{gathered}
$$

where $r$ is the amplitude coefficient of reflection, $M$ is the characteristic matrix of the system, $d$ is film thickness, and $h$ is phase difference associated with the optical path length difference.

The reflectance spectrum given in equation (1) is simplified for $\theta_{1}=0^{\circ}$ and $n_{1}=n_{3}=n_{0}$

$$
R\left(k_{0}\right)=\left[1+\left(\frac{2 n_{2} n_{0}}{n_{2}^{2}+n_{0}^{2}}\right)^{2} \sin ^{-2}\left(2 \pi k_{0} D\right)\right]^{-1}
$$

where $D=n_{2} d$ is optical thickness of dielectric film. $R\left(k_{0}\right)$ is a non-stationary signal whose repetition frequency is related to $n\left(k_{0}\right)$, therefore, the CWT method is a suitable tool to obtain the repetition frequency of the reflectance spectrum. CWT expression of $R\left(k_{0}\right)$ is given by [17]

$$
C W T(a, b)=\frac{1}{\sqrt{a}} \int_{-\infty}^{\infty} R\left(k_{0}\right) \psi_{a, b}^{*}\left(k_{0}\right) d k_{0}
$$

where $\psi_{a, b}^{*}\left(k_{0}\right)$ is the complex conjugate of the daughter (analyzing) wavelet which is acquired by adding the dilation $a$ and the translation $b$ parameters into the mother wavelet $\psi\left(k_{0}\right)$. By using the Parseval theorem, equation (3) can be rewritten to make it convenient to use the fast Fourier algorithm (FFT) [8]

$$
C W T(a, b)=\sqrt{a}\left[\int_{-\infty}^{\infty} \hat{R}\left(x_{0}\right) \hat{\psi}^{*}\left(a x_{0}\right) \exp \left(i 2 \pi b x_{0}\right) d x_{0}\right]
$$

where $\sqrt{a}$ is the normalization constant, $\hat{R}\left(x_{0}\right)$ and $\sqrt{a} \hat{\psi}^{*}\left(a x_{0}\right)$ are the Fourier transform of $R\left(k_{0}\right)$ and $(1 / \sqrt{a}) \psi_{a, b}^{*}\left(k_{0}\right)$, respectively. By using equation (4), the calculation of wavelet transform could be much faster. In this study, GMW [18], Paul and Morlet [19] were used and their analyzing wavelet forms in $x_{0}$ domain (Fourier domain), respectively, were given by,

$$
\begin{gathered}
\hat{\psi}_{\beta, \gamma}\left(a x_{0}\right)=\xi\left(a x_{0}\right) \kappa_{\beta, \gamma}\left(a x_{0}\right)^{\beta} \exp \left(-\left[a x_{0}\right]^{\gamma}\right) \\
\hat{\psi}\left(a x_{0}\right)=\frac{2^{m}}{\sqrt{m(2 m-1) !}} \xi\left(a x_{0}\right)\left(a x_{0}\right)^{m} \exp \left(-a x_{0}\right) \\
\hat{\psi}\left(a x_{0}\right)=\frac{\sqrt{2 \pi}}{\sqrt[4]{\pi}} \exp \left(-\frac{\left[a x_{0}-w_{0}\right]^{2}}{2}\right)
\end{gathered}
$$

where $\xi\left(x_{0}\right)$ is Heaviside step function, $\kappa_{\beta, \gamma}=2[(e \gamma) / \beta]^{\beta / \gamma}$ is the normalization constant of GMW, $\beta$ and $\gamma$ are degrees of freedom of GMW, $m$ is degree of freedom of the Paul wavelet, and $w_{0}$ is the nondimensional frequency, here taken to be 6 to satisfy the admissibility condition [19].

The analyzing wavelet is the most important point in the CWT method. In this context, the resolution of analyzing wavelets can be estimated. Uncertainty values of wavelets are given in Table 1. for comparison [20] 
MEASUREMENT SCIENCE REVIEW, 21, (2021), No. 2, 61-66

Table 1. Uncertainty values of GMW, Paul, and Morlet wavelets.

\begin{tabular}{|c|c|c|c|c|c|c|c|c|c|c|c|}
\hline & \multicolumn{5}{|c|}{ GMW } & \multicolumn{5}{|c|}{ Paul } & Morlet \\
\hline & \multicolumn{5}{|c|}{$\beta$} & \multicolumn{5}{|c|}{$\mathrm{m}$} & \\
\hline \multirow{5}{*}{$\gamma$} & & 3 & 5 & 7 & 10 & 1 & 2 & 5 & 10 & 15 & \\
\hline & 3 & 0.50 & 0.50 & 0.50 & 0.50 & & & & & & \\
\hline & 5 & 0.51 & 0.51 & 0.51 & 0.50 & 0.86 & 0.64 & 0.52 & 0.51 & 0.50 & 0.5 \\
\hline & 7 & 0.53 & 0.52 & 0.52 & 0.51 & & & & & & \\
\hline & 10 & 0.57 & 0.55 & 0.54 & 0.53 & & & & & & \\
\hline
\end{tabular}

\section{NUMERICAL SIMULATION}

The reflectance spectrum shown in Fig.2.b) was computed in the range of $300-1200 \mathrm{~nm}$ wavelength (in $0.8333-3.3333 \mu \mathrm{m}^{-1}$ wavenumber $\left.\left(k_{0}=1 / \lambda\right)\right)$ by using equation (2) with given parameters

$$
\begin{gathered}
n_{2}\left(k_{0}\right)=A+B k_{0}^{2} \\
A=1.5046, B=4.2 \times 10^{-3} \mu m^{2} \\
d=5 \mu m, n_{0}=1
\end{gathered}
$$

where A and B are Cauchy parameters for Borosilicate glass BK7 [21]. A theoretically generated refractive index dispersion of BK7 by using equation (8) is indicated in Fig.2.a).
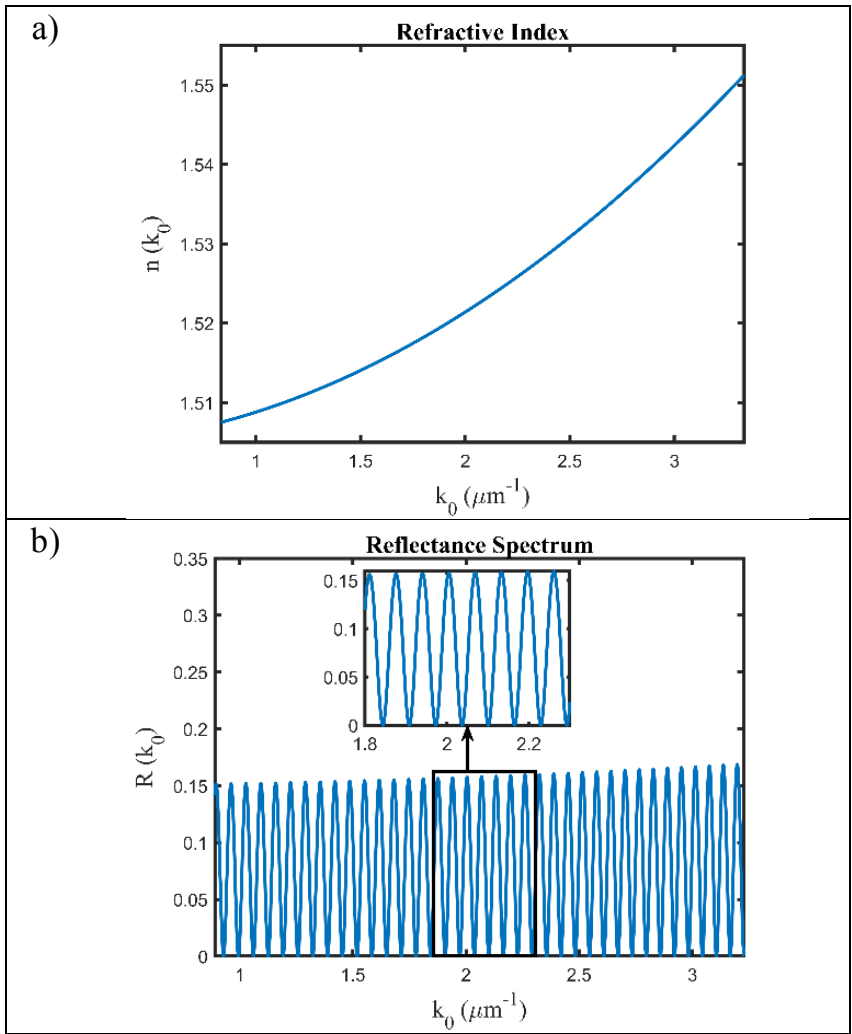

Fig.2. A theoretically generated a) refractive index dispersion of $\mathrm{BK} 7, \mathrm{~b})$ reflectance spectrum of BK7.
The reflectance spectrum shown in Fig.2.b) is analyzed by the CWT method. The power spectrum of CWT was calculated for GMW $(\gamma=3, \beta=10)$ and is plotted in Fig.3.a). The refractive index values were obtained by using GMW for $\gamma=3$ and $\beta=3,5,7,10$ and are plotted in Fig.3.b) with the comparison of the theoretically generated refractive index of BK7. This process was repeated for the Paul wavelet $(m=1$ and $m=10)$ and the Morlet wavelet. Also, the obtained values were compared with GMW $(\gamma=$ $3, \beta=10$ ) and input refractive index of BK7 and are shown in Fig.3.c). The two-term Cauchy parameters for this method were calculated from the obtained refractive index dispersions shown in Fig.3.a) and Fig.3.b) by using the leastsquares fitting method. All the determined Cauchy parameters are given in Table 2.

Table 2. The obtained Cauchy parameters of refractive index.

\begin{tabular}{lcc}
\hline & A & B \\
\hline BK7 & 1.5046 & 0.0042 \\
GMW $(\gamma=3, \beta=10)$ & 1.5046 & 0.0042 \\
GMW $(\gamma=3, \beta=7)$ & 1.5049 & 0.0042 \\
GMW $(\gamma=3, \beta=5)$ & 1.5040 & 0.0043 \\
GMW $(\gamma=3, \beta=3)$ & 1.5048 & 0.0040 \\
Paul $(m=10)$ & 1.5045 & 0.0042 \\
Paul $(m=1)$ & 1.5025 & 0.0045 \\
Morlet & 1.5042 & 0.0042 \\
\hline
\end{tabular}

The obtained refractive index dispersions shown in Fig.3.b) and Fig.3.c) indicate that the best fitted refractive index dispersion was achieved by GMW ( $\gamma=3, \beta=10)$. Also, the acquired Cauchy parameters $(A$ and $B)$ shown in Table 2 . indicate that GMW provides more accurate results with parameters $(\gamma=3, \beta=10)$.

The wavelet algorithm is functional for noisy signals due to the band-pass filter property as a nature of the wavelet transform [8]. To show the advantages of the CWT method, a noisy reflectance spectrum was simulated with adding $2 \%$ noise given in Fig.2.b) and is presented in Fig.4.a). The noisy reflectance spectrum is the same as the simulated spectrum shown in Fig.1.b). Just a random noise of $2 \%$ in magnitude was added to the reflectance signal. All calculation processes were repeated for the noisy reflectance spectrum. The calculated refractive index dispersions from the noisy 
reflectance spectrum by GMW for $\gamma=3$ and $\beta=3,5,7,10$ are indicated in Fig.4.b). Also, the noisy reflectance spectrum was analyzed by the Paul wavelet $(m=1$ and $m=10)$ and the Morlet wavelet. The results are plotted in Fig.6.c) with comparisons. Cauchy parameters obtained from noisy reflectance spectrum are given in Table 3.

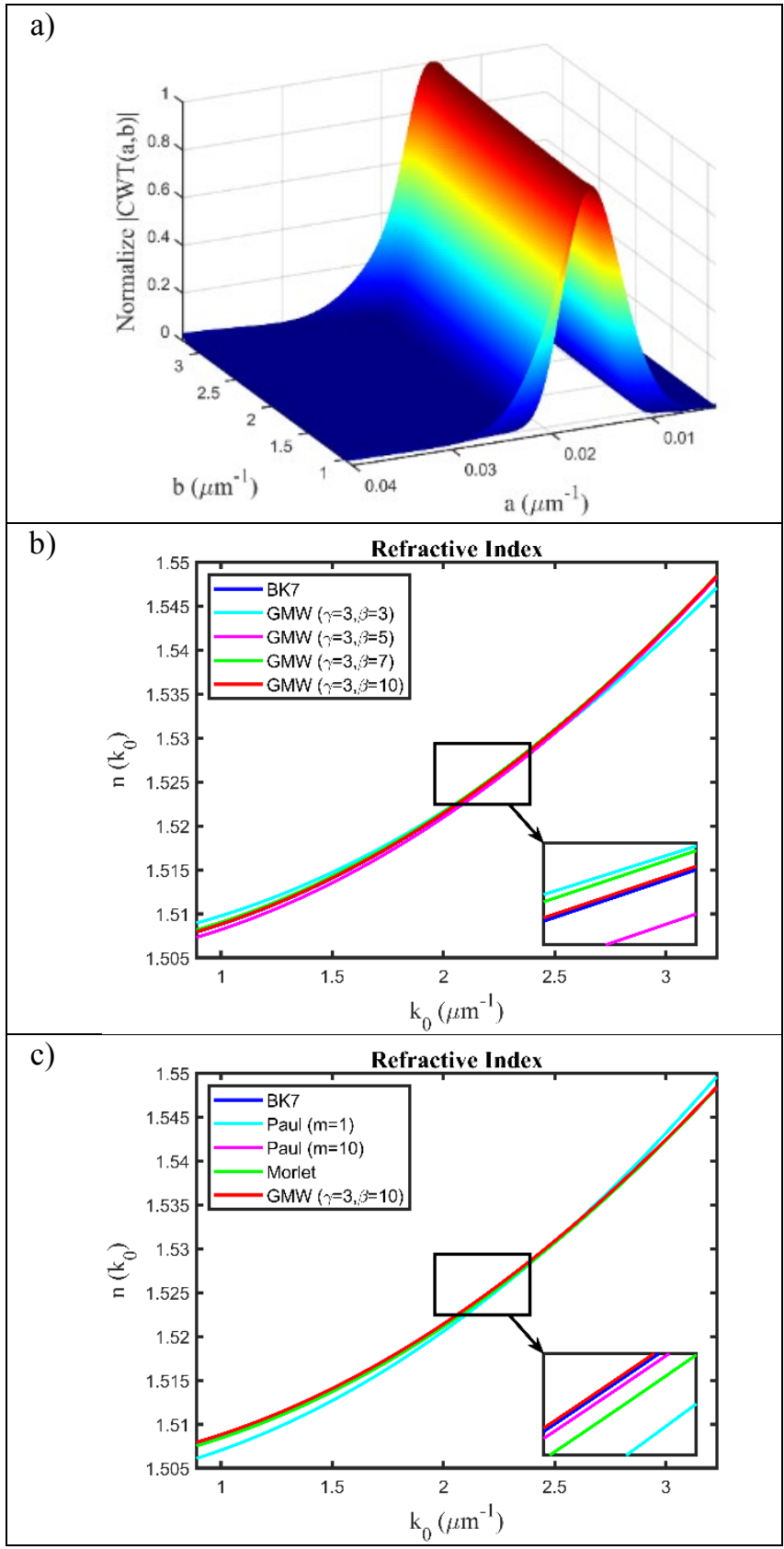

Fig.3. a) The power spectrum of $\operatorname{CWT}(a, b)$ was calculated for GMW $(\gamma=3, \beta=10)$, b) Refractive index obtained by GMW for $\gamma=3$ and $\beta=3,5,7,10$ compared with input refractive index of BK7, c) Refractive index obtained by Paul $(m=1$ and $m=10)$ and Morlet and compared with GMW for $(\gamma=3, \beta=10)$ and input refractive index of BK7.
When the results shown in Fig.4.b), Fig.4.c) and Table 3. were examined, GMW $(\gamma=3, \beta=10)$ was highly successful.

The error calculation gives another perspective to this study. The relative uncertainty for the refractive index was determined for both noiseless and noisy signals by using equation (11) and the results are given in Table 4.

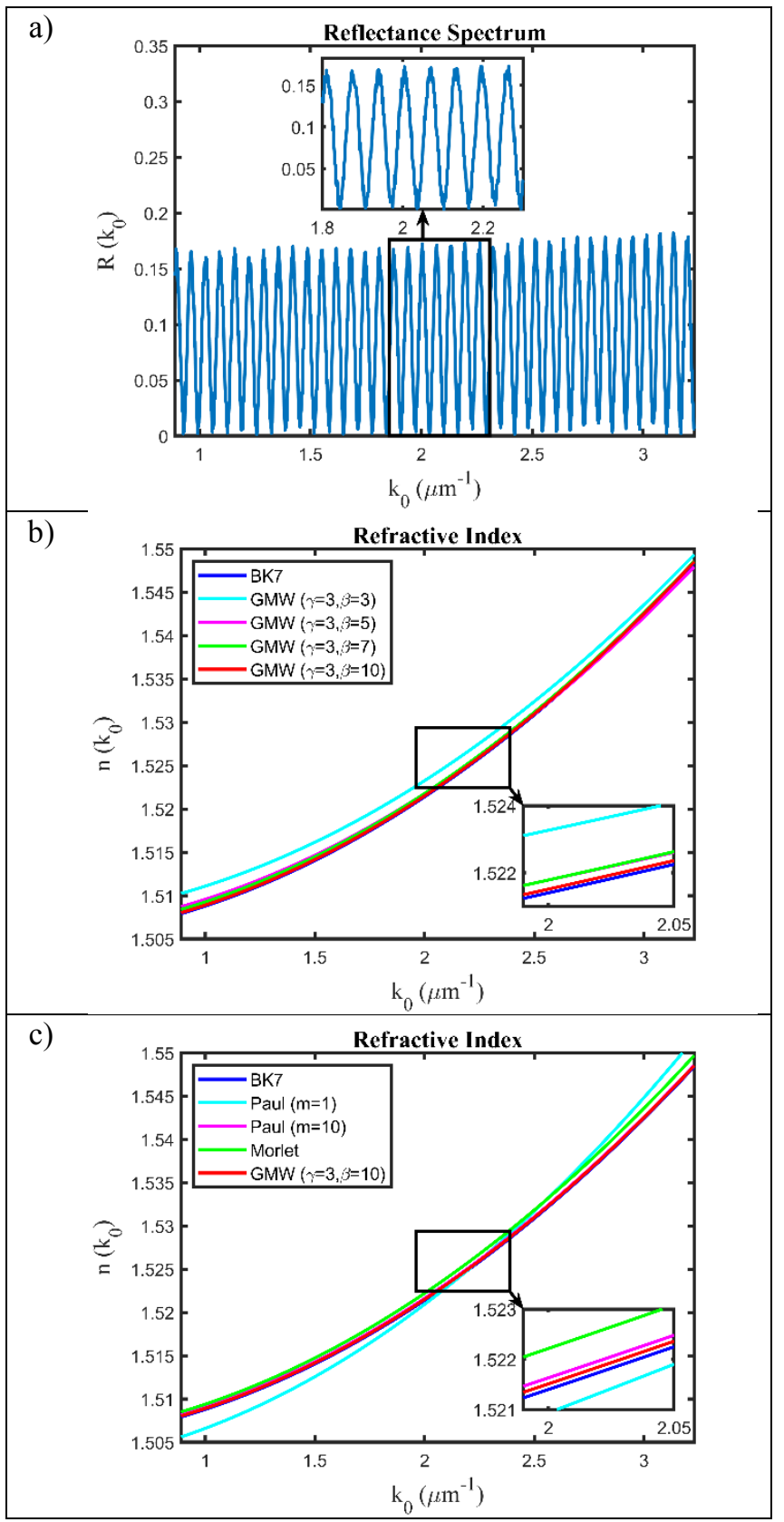

Fig.4. a) A noisy reflectance spectrum was simulated with adding $2 \%$ noise, b) The refractive index was obtained from the noisy reflectance spectrum by GMW for $\gamma=3$ and $\beta=3,5,7,10$ and input refractive index of BK7, c) obtained by Paul $(m=1$ and $m=$ $10)$ and Morlet with a comparison with GMW for $(\gamma=3, \beta=10)$ and input refractive index of BK7. 


$$
\text { Error }=\frac{\Delta n}{n}=\left|\frac{n_{\text {measured }}-n_{B K 7}}{n_{B K 7}}\right|
$$

Table 3. The obtained Cauchy parameters of refractive index from noisy reflectance spectrum.

\begin{tabular}{lcc}
\hline & A & B \\
\hline BK7 & 1.5046 & 0.0042 \\
GMW $(\gamma=3, \beta=10)$ & 1.5047 & 0.0042 \\
GMW $(\gamma=3, \beta=7)$ & 1.5051 & 0.0042 \\
GMW $(\gamma=3, \beta=5)$ & 1.5055 & 0.0041 \\
GMW $(\gamma=3, \beta=3)$ & 1.5069 & 0.0040 \\
Paul $(m=10)$ & 1.5049 & 0.0042 \\
Paul $(m=1)$ & 1.5016 & 0.0047 \\
Morlet & 1.5051 & 0.0042 \\
\hline
\end{tabular}

Table 4. The obtained relative uncertainty values of refractive index for both noiseless and noisy signals.

\begin{tabular}{lcc}
\hline & $\begin{array}{c}\text { Error } \\
\left(\times \mathbf{1 0}^{-4}\right)\end{array}$ & $\begin{array}{c}\text { Error }_{\text {noise }} \\
\left(\times \mathbf{1 0}^{-\mathbf{4}}\right)\end{array}$ \\
\hline GMW $(\gamma=3, \beta=10)$ & 0.23 & 0.73 \\
GMW $(\gamma=3, \beta=7)$ & 1.16 & 2.37 \\
GMW $(\gamma=3, \beta=5)$ & 2.63 & 2.68 \\
GMW $(\gamma=3, \beta=3)$ & 3.89 & 11.60 \\
Paul $(m=10)$ & 0.45 & 1.47 \\
Paul $(m=1)$ & 6.07 & 9.23 \\
Morlet & 1.62 & 5.68 \\
\hline
\end{tabular}

The relative error is a measure of uncertainty. The relative uncertainty values for the refractive index shown in Table 4. indicate that GMW $(\gamma=3, \beta=10)$ has minimum uncertainty for both noiseless and noisy signals.

\section{DisCUSSION / CONCLUSIONS}

In this study, GMW was improved as a new implementation of the calculation of the refractive index from the reflectance spectrum of the dielectric film. Besides, all the computations were repeated with Paul and Morlet wavelets for comparison. Also, a noisy reflectance spectrum was analyzed with all the wavelets.

The choice of wavelet has an important role in the analysis. In this context, the uncertainty principle provides convenience, because lower uncertainty means more accurate measurement. As the state of uncertainty values is shown in Table 1., in this work, the wavelets were chosen for the minimum uncertainty values around 0.5 . Morlet wavelet has minimum uncertainty value but it has a constant shape. For this reason, sometimes it cannot achieve good results. Paul has one degree of freedom which ensures limited control. Besides, GMW provides more control on the analysis with two degrees of freedom parameters when compared with Paul and Morlet wavelets. The obtained results support these impressions.

With the frame of the results indicated in Fig.3. and Table 2., GMW achieved more accuracy compared with Morlet and Paul wavelets. Moreover, the results obtained from the noisy reflectance spectrum and shown in Fig.4.b), Fig.4.c) and Table 3. approve the success of GMW.

Another factor that shows the advantages of GMW is the obtained relative uncertainty values of the refractive index for both noiseless and noisy signals.

As an advantageous aspect of GMW, it is very convenient for different applications and complex analysis with two degrees of freedom parameters

\section{ACKNOWLEDGMENT}

This work was supported by the Turkish Scientific and Technical Research Council (TUBITAK-MFAG no: 115F168). This study was evaluated from Erhan Tiryaki's doctoral dissertation.

\section{REFERENCES}

[1] Manifacier, J.C., Gasiot, J., Fillard, J.P. (1976). A simple method for the determination of the optical constants $\mathrm{n}, \mathrm{k}$ and the thickness of a weakly absorbing thin film. Journal of Physics E: Scientific Instruments, 9 (11), 1002-1004.

[2] Swanepoel, R. (1983). Determination of the thickness and optical constants of amorphous silicon. Journal of Physics E: Scientific Instruments, 16, 1214.

[3] Minkov, D.A. (1989). Method for determining the optical constants of a thin film on a transparent substrate. Journal of Physics D: Applied Physics, 22 (1), 199-205.

[4] Müllerová, J., Mudroň, J. (2000). Determination of optical parameters and thickness of thin films deposited on absorbing substrates using their reflection spectra. Acta Physica Slovaca, 50 (4), 477-488.

[5] Herrmann, P.P. (1980) Determination of thickness, refractive index, and dispersion of waveguiding thin films with an Abbe refractometer. Applied Optics, 19 (19), 3261.

[6] Nestler, P., Helm, C.A. (2017). Determination of refractive index and layer thickness of nm-thin films via ellipsometry. Optics Express, 25 (22), 27077.

[7] Shimizu, Y., Batres, R., Zhang, Z. (2007). Frontiers in Computing Technologies for Manufacturing Applications. Springer, ISBN 978-1-84628-954-5.

[8] Coşkun, E., Özder, S., Tiryaki, E. (2013). The Paul wavelet algorithm: An alternative approach to calculate the refractive index dispersion of a dielectric film from transmittance spectrum. Applied Physics B: Lasers and Optics, 113 (2), 243-250.

[9] Tiryaki, E., Coşkun, E., Kocahan, Ö., Özder, S. (2017). A simulation study for determination of refractive index dispersion of dielectric film from reflectance spectrum by using Paul wavelet. In AIP Conference Proceedings, $1815,050014$.

[10] Kocahan, Ö., Coșkun, E., Tiryaki, E., Özder, S. (2019). The zero order generalized Morse wavelet method to determine the refractive index and extinction coefficient dispersions of an absorbing film. Thin Solid Films, 673, 72-77. 
[11] Özcan, S., Coşkun, E., Kocahan, Ö., Özder, S. (2019). Simultaneous determination of the thickness and refractive index dispersion of dielectric films by the Paul wavelet transform. Thin Solid Films, 692, 137602.

[12] Padera, F. (2013). Measuring Absorptance (k) and Refractive Index (n) of Thin Films with the PerkinElmer Lambda 950/1050 High Performance UV-Vis/NIR Spectrometers. Application Note. Shelton, CT USA: PerkinElmer, Inc.

[13] Ataç, E., Dinleyici, M.S. (2020). Nanoscale curved dielectric film characterization beyond diffraction limits using spatially structured illumination. Optical Fiber Technology, 58, 102267.

[14] El-Zaiat, S. (1997). Application of multiple-beam white-light fringes for measuring the refraction and dispersion of mica. Optics \& Laser Technology, 29 (8), 495-500.

[15] Coşkun, E., Sel, K., Özder, S. (2010). Determination of the refractive index of a dielectric film continuously by the generalized S-transform. Optics Letters, 35 (6), 841.

[16] Hecht, E. (2017). Optics: Fifth Edition. Pearson, ISBN 978-0133977226.
[17] Grossmann, A., Morlet, J. (1984). Decomposition of Hardy functions into square integrable wavelets of constant shape. SIAM Journal on Mathematical Analysis, 15 (4), 723-736.

[18] Olhede, S.C., Walden, A.T. (2002). Generalized Morse wavelets. IEEE Transactions on Signal Processing, 50 (11), 2661-2670.

[19] Torrence, C., Compo, G.P. (1998). A practical guide to wavelet analysis. Bulletin of the American Meteorological Society, 79, 61-78.

[20] Yilmaz, Ö.K., Coşkun, E., Özder, S. (2014). Generalized Morse wavelets for the phase evaluation of projected fringe pattern. Measurement Science and Technology, 25 (10), 105701.

[21] Jenkins, F.A., White, H.E. (2001). Fundamentals of Optics, 4th ed. McGraw-Hill Education, ISBN 9780072561913. 